\title{
Incidentali nella prosa di Pavese
}

\author{
Claudia Bussolino e Margherita Quaglino* \\ Università degli Studi di Pavia e Università degli Studi di Siena \\ bclaudiab@gmail.com \\ qeset@yahoo.it
}

\begin{abstract}
Riassunto
L'intervento analizza tipi e occorrenze di incisi e proposizioni incidentali in quattro romanzi di Cesare Pavese: Paesi tuoi, Il compagno, Tra donne sole, La luna e i falò. Gli incisi (legati tanto all'oralità quanto alla tradizione letteraria) si prestano sia all'approfondimento soggettivo-lirico della psicologia dei personaggi, sia alla moltiplicazione dei piani e delle prospettive del racconto, sia alla stilizzazione sintattica, alla frammentazione (con introduttori grafici marcati) del tessuto della frase.

Dall'esame dei tipi (incidentali primarie; incidentali introdotte da trattino all'inglese; incisi di parola e di sintagma; incisi didascalia; incidentali di secondo grado), si nota che in Pavese gli incisi diventano uno strumento sintattico maneggevole e polifunzionale, che scioglie l'autore dalle preoccupazioni di una resa naturalistica della realtà, e gli consente di attingere, soprattutto dopo Il compagno, al potenziale della narrazione autodiegetica, con una resa sintattica veloce, segmentata, ellittica ma non confusa, in quanto condotta e tessuta sulla trama di un'oralità stilizzata e sull'ordito di simmetrie e parallelismi di stampo classico.
\end{abstract}

Parole chiave: Pavese, incidentali, incisi, romanzi, Neorealismo.

\section{Parenthetical clauses in Pavese's prose}

\begin{abstract}
The essay analyzes types and occurrences of parentheses and parenthetical clauses in four novels by Cesare Pavese: Paesi tuoi, Il compagno, Tra donne sole, La luna e i falò. Parentheses - tied equally to orality and literary tradition - fit either for deeping character psychology in a subjective lyrical manner or multiplying narrative planes and perspectives or even gaining syntactic stylization and phrasal texture fragmentation by the use of marked graphic introducers.
\end{abstract}

\footnotetext{
* L'intervento è frutto della comune riflessione e del confronto assiduo tra le due autrici; tuttavia, per fini specifici, si attribuiscono i paragrafi 1-3, 5-6, 8 a Margherita Quaglino, 4 e 7 a Claudia Bussolino.
} 
By the examination of the types (primary parentheticals; parentheticals introducted by a dash; word and syntagm parentheses; didascalic parentheses; secondary parentheticals), it may be noted that parentheses in Pavese become an easy-to-handle and multipurpose syntactic tool, that frees the author's mind from the worries of a naturalistic restitution of reality, and allows him - particularly after Il compagno - to reach the potential of autodiegetic narration, through a syntactic restitution that is fast, segmented, elliptical, yet not confusing, for it is unfolded and woven on the warp of a stylized orality and the weft of symmetries and parallelisms of firm classic mould.

Key words: Pavese, parentheticals, syntax, novels, Neorealism.

Bussolino, Claudia e Quaglino, Margherita. 2011. Incidentali nella prosa di Pavese. Cuadernos de Filología Italiana, nº extraordinario: págs. 83-108.

Sommario: Introduzione 1. Criteri di schedatura 2. Incidentali primarie 3. Trattino all'inglese 4. Incisi di parola e di sintagma 5. Incisi didascalia 6. Incidentali di secondo grado 7. Conclusioni. Bibliografia.

\section{Introduzione}

Il nostro intervento analizza tipi e occorrenze di incisi e proposizioni incidentali in quattro romanzi di Pavese: Paesi tuoi, Il compagno, Tra donne sole, La luna e i falo ${ }^{1}$. Il primo e l'ultimo rappresentano l'inizio e la fine del percorso di Pavese narratore; Il compagno, il punto di massima approssimazione alla poetica neorealista; Tra donne sole, il più compiuto esperimento di prosa narrativa relativamente alla costruzione dell'intreccio e della psicologia dei personaggi (Mutterle 1998: 200). L'analisi di incisi e proposizioni incidentali ci è sembrata un osservatorio privilegiato per verificare la portata sulla prosa pavesiana dell'ampio dibattito che fra gli anni trenta e gli anni cinquanta conduce alla decisiva affermazione del genere romanzo in Italia, da un complesso crogiolo che si alimenta sì dell'importazione sempre più massiccia di modelli narrativi extranazionali ma anche e soprattutto della forte pressione esercitata sul milieu letterario dagli eventi storici e dai mutamenti politici e sociali in corso $^{2}$. La prosa di Pavese si colloca appunto su questo crinale, tra prosa d'arte, realismo narrativo, neorealismo e questione della lingua del romanzo (Mengaldo 1992: 135-182; Mengaldo 1996; Testa 1997: 223-271; Coletti 20012003), l'impiego delle incidentali ci pare si presti a funzionare da cartina al tornasole delle soluzioni via via sperimentate e adottate dall'autore piemontese.

Anzitutto per «la duplice derivazione» degli incisi, «retorica e popolare»: gli incisi possono rimandare infatti tanto «al piano del discorso vivo» (Mortara 1956:

\footnotetext{
1 L'edizione di riferimento è quella Einaudi-Pléiade uscita nel 2000, a cura di Marziano Guglielminetti.

2 Della troppo vasta bibliografia sull'argomento si possono leggere il breve compendio di Asor Rosa (2003) e il capitolo che al romanzo italiano tra 1930 e 1950 dedica Tellini (1998: 330-388). In particolare sugli anni '40 e sugli scrittori di ispirazione neorealista Corti (1978: 25-110).
} 
4) quanto a un registro alto di tradizione letteraria. Inoltre per la loro funzione di commento, descrittiva-digressiva, gli incisi si prestano sia all'approfondimento soggettivo-lirico della psicologia dei personaggi sia alla moltiplicazione dei piani e delle prospettive del racconto quanto ancora alla spezzatura, all'imprevista deviazione sintattica, alla frammentazione del tessuto della frase ${ }^{3}$.

\section{Criteri di schedatura}

L'estensione. Abbiamo distinto incisi di parola o di sintagma da incisi di frase o proposizioni incidentali ${ }^{4}$; a parte abbiamo analizzato gli incisi con funzione di didascalie, luogo privilegiato per la verifica della tenuta «realistico-mimetica» del discorso diretto e indiretto (Coletti 1989: 55).

La tipologia sintattica. Sulle proposizioni incidentali non c'è accordo negli studi grammaticali: mentre per Borgato-Salvi il «segmento parentetico» non può intrattenere alcun «legame sintattico ${ }^{5}$ con la frase ospite, Serianni ${ }^{6}$ ammette la possibilità del legame sintattico distinguendo «tra incidentali primarie (quando la frase è priva di una congiunzione introduttiva [...]) e incidentali secondarie, quando la frase è formalmente collegata al periodo attraverso una congiunzione coordinativa $[\ldots]$ o subordinativa». Cignetti e poi Telve lasciano infine cadere la distinzione tra incisi di primo o secondo grado, valendosi di un criterio di riconoscimento principalmente topologico: qualsiasi tipo di frase può essere considerata incidentale se si trova in posizione incidentale ${ }^{7}$. In un testo letterario è evidente che una incidentale primaria introdurrà una sprezzatura maggiore e risulterà più funzionale all'inserzione di commenti metanarrativi o di frammenti memoriali, mentre l'incidentale secondaria tenderà a agglutinarsi al piano discorsivo principale aggiungendo particolari descrittivi

\footnotetext{
3 Mortara Garavelli (2003: 297-300) classifica gli «enunciati parentetici, o incidentali» tra le forme di metatassi per aggiunzione, e distingue: «Le aggiunte alla frase minima completa, che proprio perché completa può essere considerata unità massima, sono percepite come 'scarti' retorici, quando per mezzo di esse si dà rilievo, allungandoli, a elementi secondari; quando si aumenta la distanza fra elementi principali interponendone altri; quando si attira l'attenzione sul messaggio modificandone la struttura normale con accorgimenti amplificativi».

${ }^{4}$ Ferrari-Zampese (2000: 363) sottolinea il dato della variabilità dell'estensione del segmento parentetico, che, se delimitato da parentesi o trattini, può anche essere dato da «una frase complessa sintatticamente indipendente costruita per coordinazione o subordinazione» o da «un insieme di unità linguistiche, vale a dire un breve testo».

5 Borgato-Salvi (1995: 165). Secondo questi autori si può parlare di frase incidentale o parentetica per la frase esemplificata in (1):

(1) Domani - mi dicono - arriverà anche Mario.

ma non per la frase (2):

(2) Dai Rossi - se devo essere sincero - mi sono annoiato molto.

6 Serianni (2006: 625-626).

${ }^{7}$ Cignetti (2001); Telve (2008: 84-85). I criteri di riconoscimento elencati con chiarezza e precisione da Cignetti sono: la delimitazione grafica; la struttura prosodica; l'espletività; la riformulabilità. Per una panoramica esauriente dei parametri di individuazione e delle definizioni delle incidentali in ambito francofono si veda Pétillon-Boucheron (2002: 77-130).
} 
in modo sintatticamente non marcato ${ }^{8}$ : ci è sembrato utile perciò mantenere la distinzione di Serianni e concentrare l'attenzione dell'analisi anzitutto sulle incidentali primarie.

La posizione. Mentre Borgato-Salvi e Telve riconoscono in posizione parentetica parole, sintagmi e proposizioni all'interno o alla fine della frase, Serianni e con più convinzione Cignetti $^{9}$ ammettono posizione parentetica anche a inizio frase. Pur allargando lo spettro dell'analisi a incisi e incidentali collocati in tutte e tre le posizioni ammissibili, ci siamo concentrate su quelli posti all'interno di frase per il valore stilistico decisamente più marcato di cui si fanno carico nel testo letterario.

Gli introduttori grafici. Gli incisi possono essere segnalati da parentesi, trattini, virgole, punti ${ }^{10}$. Anche qui è evidente che le virgole giocano un ruolo stilisticamente più debole rispetto agli altri introduttori (Telve 2008: 85), che abbiamo perciò privilegiato nell'analisi. Tratto marcato dei romanzi della maturità di Pavese è l'uso anglosassone che prevede la presenza di un solo trattino per le incidentali poste in fine di frase. È un tratto scarsamente diffuso nel romanzo italiano di questi anni, e viene probabilmente a Pavese dalla lunga consuetudine con la narrativa anglosassone: ci è parso perciò interessante cercare di capire la funzione che assolve nella prosa pavesiana.

Infine, poiché il carattere fondamentale dell'inciso è quello di introdurre una «deviazione netta e provvisoria all'interno della struttura del periodo» (Devoto 1950: 59), non abbiamo considerato incisi le coordinate o subordinate allineate secondo procedimenti di parallelismo o disposte su diversi piani subordinativi all'interno di una frase complessa, a differenza di quanto sostenuto a esempio da Dardano nei suoi studi sulla sintassi della prosa antica ${ }^{11}$.

${ }^{8}$ Le incidentale primarie si configurano sovente infatti come elementi extranucleari con funzione di avverbiali di frase, mentre quelle secondarie rivestono di preferenza funzione avverbiale e circostanziale.

${ }^{9}$ Cignetti cita al riguardo Lonzi (1981: 363).

${ }_{10}$ Utile la precisazione di Ferrari-Zampese (2000: 365): nel caso di segmenti testuali isolati tra due virgole, la distinzione tra parentetiche e non parentetiche diventa più complessa, stabilendo due criteri di riconoscibilità. Un segmento testuale tra virgole viene considerato senz'altro parentetico quando «separa parole strettamente legate dal punto di vista sintattico, come un aggettivo e un nome, un ausiliare e il verbo cui esso si associa» o quando «il contenuto dell'espressione si lega all'atto linguistico associato alla frase che lo contiene». Si tratta di criteri condivisibili, ma che rischierebbero di escludere gran parte dei segmenti parentetici a inizio o fine frase.

${ }^{11}$ Per il primo caso si veda a esempio Dardano (1992: 331). Per il secondo caso, si veda la citazione di un passo del Cortegiano: «esempio [...] di Senocrate; che, essendo versato negli studii, astretto ed obbligato dalla profession sua (che è la filosofia, la quale consiste nei boni costumi e non nelle parole), vecchio, esausto del vigor naturale, non potendo né mostrando segno di potere, s'astenne da una femina publica». Dardano commenta che in questo brano «il grado di stilizzazione è dimostrato dal rapido succedersi di incidentali di diversa natura (un gerundio, due participi passati, due relative tra loro legate, un aggettivo, un participio passato, due gerundi), le quali, nel loro insieme, compongono un dettato breviloquente, d'impronta latineggiante» (Dardano 1992: 459-60). 


\section{Incidentali primarie}

Colpisce anzitutto la forte escursione di frequenza di incisi e incidentali con introduttori grafici marcati - trattino inglese, due trattini e parentesi - tra Paesi Tuoi e gli altri tre romanzi, dove sono via via più numerosi: quattro in tutto invece nel primo romanzo pubblicato da Pavese, in corrispondenza delle quattro sole incidentali primarie presenti nel testo, tutte e quattro all'interno di frase:

Ma quel Berto (si chiamava come me) che quei paesi li aveva girati e si maneggiava Talino da buon maniscalco, tirava a ficcargli in testa che adesso stesse tranquillo (PT 14).

Per strada - era già sera - le raccontai come avevo imbarcato Talino (PT 9).

Di là si vedeva la prima collina, bruciata e pelata - erano tutte vigne - e il capezzolo in punta che faceva piacere guardarlo (PT 31).

La vecchia manda Gisella a prendere l'acqua dal pozzo - un buco dentro il muro, l'unico posto fresco - e volevo aiutarla a girare il mulinello ma Gisella non vuole e si attacca al mulinello e si tira su il secchio (PT 31).

La prima incidentale assolve la funzione classica di glossa o commento isolando e tematizzando il soggetto della frase già sottolineato dal deittico. Le altre introducono una digressione descrittiva: interessante soprattutto l'ultima, che accentua il cambio di soggetto nella sequenza paratattica, evidenziato anche dal cambio di tempo verbale. Secondo Corrado Grassi la variazione di soggetto e tempo verbale all'interno di strutture polisindetiche è una spia del «distacco netto» della prosa di Paesi tuoi «da quella che poteva essere considerata una semplice adesione ad un modello d'uso vivo». L'impiego di incidentali primarie coincide dunque con procedimenti che tendono a innalzare il registro linguistico: verificano questa ipotesi sia le limitate occorrenze in Paesi tuoi (romanzo nel quale «mai come altrove [...] Pavese è vicino a un concreto modello dialettale e gergale» (Grassi 1964: 63-64)) sia il confronto con le prose precedenti a Paesi tuoi, nelle quali secondo Mutterle «l'uso [...] di parentetiche e incidentali che spezzano continuamente la linearità della sintassi $[\ldots]$ ha provenienza di origine colta e finemente letteraria» ${ }^{12}$. Se a questo si aggiunge che «in Paesi Tuoi il presente costruisce il tempo esteriore, serve all'oggettività narrativa, mentre l'imperfetto è delegato a inserirci nel tempo interiore, psicologico o in quello diegetico» (Corti 1978: 88), ne risulta che l'incidentale primaria vale da tramite in questo delicato passaggio tra $i$ due piani discorsivi presenti nel romanzo ${ }^{13}$.

12 Mutterle (1969: 123-124). In Ciau Masino l'uso degli incisi marca la distanza tra linguaggio autoriale e linguaggio dei personaggi (Mutterle 1970: 578).

13 Anche incidentali secondarie possono assolvere a questa funzione: «E accendevo e fumavo e la stoppia era un po' bianca e un po' nera, secondo che andava la luna, e si levò il vento e le canne scricchiolavano forte coi grilli, e tutti i cani dormivano» (PT 41); «Volevo chiedere se quel sole era lo stesso di Monticello, volevo parlare, volevo entrare in quel caffè del biliardo e dormire, ma camminavo e la stazione, da piccola ch'era in principio, ci viene incontro, si fa come un portico, e usciamo sotto la tettoia, mi butto sulla panca e sto zitto» (PT 15); anche incidentali di parola e sintagma: «Scendevo e salivo una fredda scala senza ringhiera, ingombra di scope e di barattoli, e l'odore della calce - un odor vivo, di montagna - mi dava alla testa» (TDS 690). 
Nel Compagno e in Tra donne sole non sono frequenti i casi di incisi che evidenzino il cambiamento di soggetto o di tempo verbale:

Tante sere ero stato con lui - la chitarra suonava e ci piaceva a tutti e due - bevevamo un bicchiere, poi si tornava lui sul corso, io nel negozio. (CO 151) In un locale sotto via Roma, vicino alla piazzetta delle Chiese, Morelli finse di contrattare cocaina col batterista, erano grandi amici, bevemmo un cocktail che ci offrirono (TDS 706, uno dei rari casi di incidentale primaria introdotta da virgole).

Quando uscimmo - volle venire a ogni costo anche lui - mi accompagnarono in macchina all'albergo (TDS 708).

Sono invece molto frequenti dopo Paesi tuoi le incidentali primarie in posizione interfrasale senza cambiamento di soggetto né di tempo verbale nella frase ospite, con casi marcati di soggetto diverso dell'incidentale rispetto alla principale soprattutto nella Luna:

Io scambiai qualche parola con la figlia - mi guardava compiaciuta -, le chiesi della sorella (TDS 711).

Questo si divincolò in segno di saluto - era un lungo biondo diplomatico -, poi subì uno strattone (TDS 685).

Quando fu uscito, mi sfilai l'abito (non mi tolsi nient'altro) e mi riavvolsi nella coperta (TDS 721).

Nel tempo che ci sedemmo sulle poltrone (Momina buttò via le scarpe) e il cameriere depose il vassoio sul tavolino, nessuno parlò e io sentivo che gli occhi ossuti di Rosetta si empivano di lacrime (TDS 731) ${ }^{14}$.

Mentre piangevo per la capra, arrivò il parroco - aveva un grosso ombrello grigio e le scarpe infangate - e mi guardò di traverso (LF 830).

A me piaceva quel cortile così grande - ci si stava in tanti e nessuno ti cercava e poi era vicino allo stradone, sotto il Salto (LF 829).

Entrai nell'aia (di nuovo il cane si avventò), dissi ch'io su quell'aia c'ero stato bambino (LF 798).

Ma intanto andò che il sor Matteo prese di petto quell'Arturo - la storia si seppe da Lanzone che passava per caso sotto il portico - e gli disse che le donne sono donne e gli uomini sono uomini (LF 862).

I due uomini lavoravano forte, sfiancavano i buoi e le donne; la più giovane era morta in un campo ammazzata dal fulmine, l'altra, Angiolina, aveva fatto sette figli e poi s'era coricata con un tumore alle costole, aveva penato e gridato tre mesi - il dottore saliva lassù una volta all'anno -, era morta senza nemmeno vedere il prete $(\mathrm{LF} 815)^{15}$.

Sono anche frequenti dopo Paesi tuoi incidentali tra principale e subordinata (o viceversa); è un caso quasi assente nel Compagno, dove la misura paratattica prevale con più evidenza:

\footnotetext{
14 In questo caso, la parentetica è inserita all'interno di una subordinata temporale.

15 L'inciso qui interrompe una sequenza paratattica insistita.
} 
Quando alla fine ebbi capito di cosa si trattava - una ragazza strillando più forte degli altri si mise a spiegarmelo - feci finta di nulla e sorrisi impassibile (TDS 693) Parlò, senza sorridere, di un salotto di Torino - disse il nome, nobiltà - dov'era successo che certi signori importanti, in attesa della padrona di casa, s'erano spogliati (TDS 681).

Ma c'eravamo appena tolta la pelliccia e bagnate le mani (Momina nella borsetta portava crema e profumo) che s'aprì la porta (TDS 721).

Per lasciarsi toccare - avevamo una stanza di un vicolo di Oakland - voleva esser sbronza. (LF 789).

Guardando verso Canelli (era una giornata colorita, serena), prendevo in un'occhiata sola la piana del Belbo, Gaminella di fronte, il Salto di fianco, e la palazzina del Nido, rossa in mezzo ai suoi platani, profilata sulla costa dell'estrema collina. (LF 812).

Quando fui stufo di ascoltare i grilli - anche lassù c'erano i grilli - scesi dal biroccio e mi feci alla porta. (LF 865).

Ma quando a mezzanotte entrai fra le carrozze in quel cortile - vista da sotto la palazzina era enorme e sulle finestre passavano ombre d'invitati - nessuno si fece vivo e mi lasciarono in mezzo ai platani un pezzo. (LF 865).

o in posizione intrafrasale:

Dopo la prima contentezza e la sorpresa, che non bastarono a darle colore (era uscita dal banco, e ci eravamo portate sull'uscio, per meglio vederci), discorremmo così, festeggiandoci (TDS 709) ${ }^{16}$.

Di nuovo mi stupì quella Nene, scultrice o pittrice che fosse, labbra grosse e frangetta, e il suo modo sfacciato di ridere come una bambina (TDS 744).

In generale dopo Paesi tuoi Pavese accentua la sperimentazione sulle potenzialità segmentanti dell'inciso. Ad esempio l'inciso può intervenire a spezzare una sequenza trimembre:

Parlammo un poco di Torino e di Roma - mi guardava stringendo gli occhi nel fumo - delle case che non si trovano, di quel nuovo caffè (TDS 704).

o addirittura a separare il soggetto dal verbo:

La gente - erano pochi, sparsi in tutta la sala - aspettava la musica, e quattro o cinque giovanotti e ragazze accaldate battevano a quell'altra il tempo sui tavoli. (CO 195). Il fatto è che Cinto - come me da ragazzo - queste cose non le sapeva, e nessuno nel paese le sapeva, se non fosse qualcuno che se n'era andato (LF 812).

o il verbo dall'oggetto:

Amelio fece - caso strano - una risata, ma solo il gesto della faccia, non la voce. (CO 168).

${ }^{16}$ La parentetica viene inserita all'interno dell' incidentale secondaria descrittiva. 
o da uno dei suoi argomenti:

Questa volta fui io che a Linda - la notte in collina - chiesi perché non facevamo qualche cosa (CO 158).

In questi ultimi esempi gli incisi convergono su un modello di sintassi isolante, di registro alto. Si inserisce in questa tendenza anche la «particolare collocazione» in incisi a fine frase di aggettivi in funzione predicativa, distanziati dal sostantivo a cui si riferiscono, frequente in Paesi tuoi e nel Compagno ${ }^{17}$ :

Poi ti aspetto in fondo al prato, sola (PT 41).

C'era ancora quel catino sul tavolo, rosso (PT 75).

L'effetto di forte mise en relief rimanda ai due elementi fondamentali dell'intreccio di Paesi tuoi: la relazione tra il protagonista e la giovane Gisella e l'assassinio di quest'ultima.

Un bel momento alzo la testa e vedo Linda sulla porta, col dito sul labbro, contenta (CO 159).

Gina ci accolse sulla porta, contenta (CO 262).

Vedo ancora Giulianella che si schiaccia la cicca, nervosa (CO 270).

In Tra donne sole è poco frequente questa costruzione, sostituita da altre meno marcate, come quella priva di iperbato:

Morelli, testardo, parlava di Capri (TDS 707).

Gisella mi squadrava, sospettosa, con un mezzo sorriso d'invito (TDS 709).

o quella con avverbiale bimembre e impiego espressivo-intonativo della virgola:

Buttò il tovagliolo e se ne andò, grasso e solenne (TDS 682).

Si misero a un tavolo, serie serie (TDS 719).

Parlò dei pittori giovani di Torino, con intenzione e ingenuità (TDS 752).

Pur collaborando alla persistenza di una sintassi isolante, di tradizione letteraria, gli incisi veicolano (nei diversi piani discorsivi) frammenti di discorso diretto o indiretto di più immediata prossimità al registro colloquiale:

Mi disse dei nomi, lasciò intendere che non era tutta gente per bene, che la gioventù s'era inquinata e s'inquinava - non faccio questione di ceto, perbacco - ma

17 Cfr. Matarrese (1973: 288). Secondo Mengaldo il «persistere di una sintassi "isolante" [...] in presenza di un materiale lessicale volutamente abbassato a livelli più mediocri» è uno degli esempi più tipici del «passaggio dalla civiltà del frammento e del capitolo alla nuova narrativa» (Mengaldo 1996: 169): a conferma del fatto che il primo Pavese si colloca in questa posizione intermedia, di ricerca, tra le forme della prosa d'arte e l'incipiente ritorno alla narratività. 
dopo la guerra, e anche prima, chi ci capiva più niente? (TDS 696).

Le dissi che fin che una donna arrossisce non è ancora il caso di parlare di stima. (Lei si scusò. Disse: - Arrossisco per niente -). Le dissi che tutto va bene finché non fa male alla salute.

Io suonavo - Pablo qui, Pablo là - ma non ero contento, mi è sempre piaciuto suonare con qualcuno che capisca, invece quelli non volevano che gridare più forte (CO 149).

Di Nuto musicante avevo avuto notizie fresche addirittura in America - quanti anni fa? - quando ancora non pensavo di tornare, quando avevo mollato la squadra ferrovieri e di stazione in stazione ero arrivato in California (LF 789).

Mi tornarono in mente Gisella e le figlie; il negozietto, - ci siamo ristrette, - e tutto per tenerle a far niente, nel velluto (TDS 724).

Con frequenti inserzioni di tratti dell'oralità:

La notte che Amelio si ruppe la schiena sulla strada di Avigliana, ero andato con tre o quattro a una merenda in collina - mica lontano, si vedeva il ponte - e avevamo bevuto e scherzato sotto la luna di settembre, finché per via del fresco ci toccò cantare al chiuso (CO 149).

Le figlie - ne aveva due, Rosa e Lina: una tossiva, era anemica, l'altra no, quindici anni, tutte e due studiavano - erano un grosso guaio (TDS 710).

Notevoli nella Luna e i falò gli inserti di discorso diretto in inglese: sia perché il periodo trascorso in America è fondamentale per il personaggio Anguilla, sia perché la funzione di questi inserti si avvicina a quella di glosse che traducono nel presente l'esperienza del passato:

Vivevano sole, chi nelle fabbriche delle conserve, chi in ufficio - Rosanne era una maestra ch'era venuta da chissà dove, da uno stato del grano, con una lettera per un giornale del cinema, e non volle mai raccontarmi che vita avesse fatto sulla costa. Diceva solo ch'era stata dura - a hell of time (LF 854).

Ma per lei una sola cosa contava - decidermi a tornare con lei sulla costa e aprire un locale italiano con le pergole d'uva - a fancy place, you know - e il cogliere l'occasione che qualcuno la vedesse e le facesse una foto, da stampare poi su un giornale a colori - only gimme a break, baby (LF 854).

Come si fosse messa in mente ch'io potevo servirle non so; quando le chiedevo perché veniva a letto con me, rideva e diceva che dopo tutto ero un uomo (Put it the other way round, you come with me because I'm a girl). [...] Non toccava una goccia di liquore (you looks, you know, are your only free advertising agent) e fu lei che, quando abolirono la legge, mi consigliò di fabbricare il prohibition-time gin, il liquore del tempo clandestino (LF 854).

Ma a fronte di queste intersezioni tra incidentali, discorso diretto e forme dell'oralità, soprattutto nella Luna le incidentali interagiscono con strutture anaforiche che rimandano al contrario a una prosa controllata, preziosa:

A casa dissi che ci andavo per lavoro, per conoscere gente - qualcuno voleva sentirmi - e la chitarra è questione di farsi sentire (CO 181). 
La collina di Gaminella, un versante lungo e ininterrotto di vigne e di rive, un pendìo così insensibile che alzando la testa non se ne vede la cima - e in cima, chi sa dove, ci sono altre vigne, altri boschi, altri sentieri - era come scorticata dell'inverno, mostrava il nudo della terra e dei tronchi (LF 782).

Ho pensato sovente che razza di figli sarebbero potuti uscire da noi due - da quei suoi fianchi lisci e duri, da quel ventre biondo nutrito di latte e di sugo d'arancia, e da me, dal mio sangue spesso (LF 855).

Chi non lo voleva era Irene, perché diceva ch'era un uomo falso - che la musica non l'ascoltava neanche, che a tavola non sapeva stare, e faceva giocare Santina soltanto per ingraziarsi la madre (LF 860: struttura trimembre con variatio di dislocazione a sinistra).

Occorre sottolineare che da questi esempi emerge inoltre una continuità sintattica anomala tra inciso e frase ospite che snatura la caratteristica principale dell'inciso stesso, quella cioè di evidenziare una devianza, una rottura, un a parte rispetto alla frase entro cui è inserito. Esempi eclatanti di questa anomalia dopo Paesi tuoi sono i casi nei quali viene meno l'accessorietà sintattica dell'inciso:

I cani continuavano a urlare, in quel mare grigio ch'era la pianura - una voce che rompeva l'aria come il canto del gallo - metteva freddo e disgusto (LF 818).

Così venne l'inverno e cadde molta neve e il Belbo gelò - si stava al caldo in cucina o nella stalla, c'era soltanto da spalare il cortile e davanti al cancello, si andava a prendere un'altra fascina - o bagnavo i salici per Cirino, portavano l'acqua, giocavo alle biglie coi ragazzi (LF 831).

Come si vede, la seconda parte della frase ospite non è sintatticamente ammissibile se non come coordinata della frase in inciso. Nel caso di incisi di parola o sintagma, la seconda parte della frase ospite è accordata con il soggetto della incidentale:

Le dissi che noi - cioè io - avrei voluto salutarla (TDS 697).

Chi restava, come Gisella, non le importava più di noi (TDS 711).

Nel secondo esempio, marcato rispetto al primo, la segmentazione della frase è accentuata dall'inserzione dell'incidentale e il pronome ridondante 'le' è accordato al soggetto dell'inciso mentre l'ellissi della preposizione ('A chi restava') tende all'anacoluto. Se assumiamo che l'incidentale rappresenti per Pavese un tratto di registro alto, questi esempi vanno nella direzione del meticciamento (discorso diretto, indiretto, forme dell'oralità) e della reinterpretazione (scambi lessicali e legami sintattici non accessori con la frase ospite) del modulo parentetico che assume valenze plurivoche.

\section{Trattino all'inglese}

Un caso ancora differente di indebolimento dell'accessorietà sintattica dell'inciso è rappresentato dall'impiego del trattino singolo all'inglese, che interessa incidentali 
primarie e incisi di parola e sintagma: pressoché assente in Paesi tuoi è un tratto che si trova spesso in concomitanza con altri marcatori di parenteticità. Due esempi di queste occorrenze multiple possono servire ci pare a distinguere valore sintattico e funzione del trattino singolo per contrasto con gli altri segni interpuntivi:

Mi disse che avevo dei pregiudizi - uno solo, ma grosso: credevo che lavorare e farsi strada, o anche soltanto lavorare per vivere, valesse le qualità - qualcuna scema, d'accordo - della gente che nasce bene.

Cercai le facce - erano le solite, chi sporgeva le labbra, chi pareva ghignasse. Colsi l'occhio della mia cameriera - stava correndo dietro la barella. Sulle voci sommesse del crocchio (c'era pure una signora in pelliccia e si torceva le mani) si levò quella di un dottore - uscì dalla porta asciugandosi le mani - e dichiarò ch'era finito (TDS 677-678).

Gli esempi confermano anche per l'inciso introdotto da trattino semplice le fondamentali funzioni di correctio, descrizione e commento: la continuità sintattica tra frase ospite e inciso però, tale che si potrebbe quasi in ogni occorrenza sostituire il trattino con i due punti, indica una minore forza frammentante rispetto a quella esercitata dai due trattini o dalla parentesi. Al tempo stesso l'abbassamento di tono caratteristico anche di questi incisi lavora in direzione della profondità prospettica; l'incassatura tonale evidenzia in genere un passaggio logico quando non avverte di uno scarto semantico o dell'inserzione di frammenti di discorso diretto o indiretto, che collaborano alla moltiplicazione dei piani discorsivi ma non subordinativi. L'effetto è quello non solo di alleggerimento dell'intelaiatura sintattica ma anche di svelamento e addizione di un secondo o terzo risvolto del reale, parallelo e non secondario (a differenza di quanto avviene per incisi introdotti da altri segni interpuntivi) a quello narrato ${ }^{18}$ (va perciò nel senso della narratività, non della lirica):

Non mi tornò il batticuore - troppe volte ci avevo pensato (CO 252).

Spalancai le persiane - sporgendo la testa c'era un cielo già chiaro (CO 255).

18 Sono soprattutto le incidentali all'inglese de Il mestiere di vivere (cito dalla seconda edizione condotta sull'autografo, Einaudi 2000) che suggeriscono per il trattino inglese questa funzione addizionale di un aspetto parallelo, speculare (non secondario, non digressivo) all'asse portante della riflessione, sottraendo al dettato assolutezza e univocità: «Non è da dimenticare che Dio significa pure cataclisma tecnico - simbolismo preparato da anni di spiragli»; «Magnolie abeti, tassi, cipressi, limoncelle - verdi cupi, metallici bronzei contro il cielo azzurro»; «Non vi si chiarisce una responsabilità, si constata un fatto - un destino»; «Capirei trasformarle [le colpe] in virtù, in modi energici, ma no - si scopre il trauma che fa sì che hai paura, per esempio, dei ranocchi e allora aspetti la guarigione»: «La bestiola che fuggiva nel grano era lo spirito - fondevi l'ancestrale e l'infantile»; «Si ha pietà di tutti - meno di quelli che si annoiano» (296; 306; 314; 319; 320). Pochi esempi di incisi introdotti da altri segni interpuntivi mi sembra bastino per verificarne al contrario il valore descrittivo-digressivo, accessorio rispetto al piano principale, semantico e sintattico, della riflessione: «Che dire se un giorno le cose naturali - fonti, boschi, vigne, campagna - saranno assorbite dalla città e dileguate, e s'incontreranno in frasi antiche?»; «Con gli altri - anche con l'unica persona che erge - bisogna sempre vivere come se cominciassimo allora»; «E allora vedrebbero che - tolte le pigrizie, tolti gli interessi inconfessati di ciascuno (vita comoda, meditazione indeterminata, sadismi eleganti) - non esiste istanza in cui diano risposta diversa da quella collettiva del P.C.»; "Il massimo dei contrasti è fra l'inconscio e il conscio (esigenze sociali, comunicative, ecc.)» $(301 ; 310 ; 313 ; 337)$. Per alcune puntualizzazioni sull'uso del trattino si veda Antonelli (2008: 203-207). 
La ragazza era entrata in albergo al mattino - veniva sola da una festa, da un ballo (TDS 679).

Ma non conoscevo Mariella e la sua tenacia - era nipote della vecchia del sofà (TDS 693).

Sapevo ch'era vedovo, gli era morta la moglie nella cascina prima di questa e dei figli i più vecchi erano morti in guerra - non gli restava che un ragazzo e delle donne (LF 796).

Qui più nessuno mi parlò delle cinque lire del municipio, l'anno dopo non pensavo più a Cossano - ero Anguilla e mi guadagnavo la pagnotta (LF 833).

Con inserzione di discorso diretto:

Mi chiesi se proprio l'avevo vista su quella barella - quanti giorni fa? quindici, venti? (TDS 715).

Con inserzione di discorso indiretto:

Nemmeno per la Madonna d'agosto Nuto ha voluto imboccare il clarino - dice che è come nel fumare, quando si smette bisogna smettere davvero (LF 792).

Un certo Pegi, che quell'inverno aveva spalato la neve sui viali - per ingaggiarsi diceva lui, per eccentricità dicevano loro (TDS 693).

Vociavano come tori su non so che questione - se nella vita si è se stessi o si deva recitare (TDS 696).

Con inserzione di discorso indiretto libero:

Gli dico che dormivano ancora tutti, ma io ero in piedi per sbrigare la macchina e andare a Monticello - c'era bene una fabbrica della luce? (PT 29).

Rividi le facce ambiziose: facce pallide, segnate, convulse - ce n'era qualcuna che si fosse distesa in un'ora di pace? (TDS 677).

Quando ci stavo io, c'era il camino che non tirava più - l'avevano poi rotto quel muro? (LF 803).

Pasticciai intorno al motore - niente da fare, non avevo bobine di ricambio. (LF 817).

Con inciso interno al discorso indiretto:

Poi dissi alla Bionda che tanto valeva far fuori le selle - nessuno di noi le sapeva aggiustare (CO 226).

Una volta mi tenne sull'uscio a parlare e non piangeva, non alzò la voce, non voleva che Amelio sentisse - mi diceva che già da bambino suo padre l'aveva battuto una volta e lasciato per morto perché scappava in bicicletta chissà dove, e che Amelio era stato malato di mal di capo per mesi e un dottore l'aveva guarito facendogli una puntura, proprio in quella cucina (CO 149: con paratassi protratta, alternado segmenti collegati per asindeto e per polisindeto).

Mi colpì il tono astioso con cui disse che Carlotta aveva fatto la sua strada - era stata ballerina in Germania durante la guerra, più nessun l'aveva vista (TDS 710). Mi dissero di tornare a riprenderle a mezzanotte, di entrare nel cortile del Nido - 
col buio gli invitati non avrebbero visto che i cuscini del biroccio erano scrostati (LF 865).

A commento dei fatti narrati:

La sciocchezza, l'allegra incoscienza di Guido quando aveva creduto di portarmi con sé e mantenermi - sapevo già tutto fin da principio (TDS 691: commento). Mi lasciai sorprendere - ero stufo di prevedere e di correre, e ricominciare l'indomani (LF 817).

Anche le incidentali all'inglese ammettono iterazioni, parallelismi, strutture elencative condividendo con gli altri tipi di incidentali già esaminati la natura di segno ambiguo, prezioso e mediocre: elemento catalizzatore a un tempo di frammentazione e di continuità:

Ogni tanto qualcuno picchiava alla spia - c'era il pane, la spesa, la conta, il bidone (CO 272).

Cosa avrei dato per avere un lavoraccio anche sporco - il facchino magari, gli altiforni di Cogne, il marinaio che gli tocca la burrasca (CO 274).

Capivo che da ragazzo, anche quando facevo correre la capra, quando d'inverno rompevo con rabbia le fascine mettendoci il piede sopra, o giocavo, chiudevo gli occhi per provare se riaprendoli la collina era scomparsa - anche allora mi preparavo al mio destino, a vivere senza una casa, a sperare che di là dalle colline ci fosse un paese più bello e più ricco (LF 806).

Per lo meno, giocando non c'è differenza - nati bene o nati male, puttane, borsaioli, fessi o furbi, tutti vogliono la stessa cosa (TDS 718).

Poteva succedergli come succede in quella valle a tanti - di venir su come una pianta, d'invecchiare come una donna o un caprone, senza sapere che cosa succede di là da Bormida, senza uscire dal giro della casa, della vendemmia, delle fiere (LF 806).

\section{Incisi di parola e di sintagma}

Anche la gamma delle soluzioni stilistiche con inserzione di incisi di parola e sintagma è in realtà vastissima. Si va dalla simulazione di inciso minimo, dovuta all'uso straniante, intonativo-segmentante della virgola:

Anche Loris fumava, una pipa, e tutti e due immusoniti (TDS 698).

al breve inciso che corrisponde a una frase monorematica a conclusione di un periodo bipartito o nel quale l'iterazione di incisi in costrutti trimembri ne diluisce la portata segmentante:

Più mi convinco che far parole non serve, più mi succede di parlare. Specialmente fra donne (TDS 677).

Poi, con la smania di uscire, di vedere, di correre per Torino, con le prime scap- 
pate nei vicoli insieme a Carlotta e alle altre, col batticuore di sentirci per la prima volta inseguite, anche quest'innocenza era finita. Strana cosa (TDS 677).

Gli incisi di parola e sintagma possono rivestire funzione di avverbiali: posti all'interno di frase, sottolineano anche qui gli snodi sintattici:

Finalmente arriva il treno, adagio, che sembrava Talino quando traversa una strada (PT 16).

Tenevano il mastello, una mano per una, e correvano piegate (PT 21).

Intanto tuona, sempre più forte, e cominciano a volare le foglie (PT 56).

Mariella, dietro, non parlava. La posammo davanti al cancello, sul suo viale (TDS 703).

Mi disse di accompagnarle fino a casa, due passi (TDS 760).

Sovente in inciso è precisato il tempo o il luogo ai quali la narrazione si riferisce, con varie interferenze di costrutti propri dell'oralità:

Questa volta fui io che a Linda - la notte in collina - chiesi perché non facevamo qualche cosa (CO 158).

Non appena fui solo con lei - l'indomani per strada - glielo chiesi (CO 198).

Ripensai l'ultima volta ch'ero stata a Torino - durante la guerra - l'indomani di un'incursione (TDS 677).

Sono avvezza a sentirne, nel nostro negozio, scandali e pettegolezzi di tutta Roma (TDS 700) ${ }^{19}$.

Padrino era morto vecchio vecchissimo - pochi anni fa - su una strada, dove i mariti delle figlie l'avevano buttato (LF 815).

Ci fu un mese - c'eran le lucciole, era giugno - che tutte le sere si vedevano spuntare da Canelli (LF 862).

Tra avverbiale e circostanziale è l'occorrenza sempre in inciso di particolari descrittivi:

Adesso era scuro e, tra grilli e cani, dalla collina non si sentiva più altro (PT 25). C'era un cane, lontano lassù, che abbaiava (PT 26).

Ieri, al buio, doveva essere così (TDS 734).

con impiego frequente del costrutto 'con + un particolare della figura' ${ }^{20}$, all'interno o a fine frase, che va nel senso di una resa rapida, scorciata e anche ritmica dei particolari descrittivi:

19 In questo esempio si notino le virgole che frammentano in modo insistito, la dislocazione a destra con ridondanza pronominale e l'ellissi della preposizione partitiva che accentua la segmentazione tra prima e seconda parte della frase.

${ }^{20}$ Coletti (2001-2003: 325-326) fa risalire l'affermazione e diffusione di questo costrutto alla prosa del Mastro Don Gesualdo, nel quale Verga mette a punto «una sintassi fortemente nominalizzante, elencativa, con forme verbali indefinite (i gerundi ad esempio), particolarmente adatta per descrivere paesaggi, personaggi, situazioni»: forma succedanea di questo schema sintattico sarebbe la forma «con + un particolare della figura», che esalta «ulteriormente l'avanzata del nome, la ritirata del verbo, giusta una traccia linguistica che sarà poi caratteristica di tutto il Novecento». 
Si asciugava il sudore, con una faccia da richiamato, e mordeva un pezzo di pane e formaggio (PT 10).

Talino si era messo subito a dormire, con la testa sul fagotto e il fazzoletto per traverso, e mi strizzava l'occhio (PT 11).

Arriva il carro nel cortile, con Gisella e Miliota sul fieno, e gettava un'ombra lunga (PT 32).

Per un po' è solo notte, coi lampi e coi grilli, poi di colpo si leva una voce di là dal cortile, da matto o da donna, che fa un urlo lungo e finisce (PT 55) ${ }^{21}$.

Noi grandi, seduti intorno a un sofà, ascoltavamo una vecchia permalosa, col nastro al collo e la mantellina di velluto, raccontare non so che storia (TDS 691). Allora mi disse, con un tono che un tempo ci avrebbe fatte ridere tutte e due, se volevo comprare (TDS 709).

Di tanto in tanto lei mi parlava così, con un sorriso da bella ragazza, e in quel momento mi pareva di non esser più un servitore (LF 885).

Frequente in Tra donne sole anche il modulo con preposizione 'in':

Entrò con un sorriso incerto, nel suo leopardo (TDS 733).

Becuccio, nella sua giacca a vento, mi guidò deciso ai tavolini di fondo (TDS 758).

e anche più frequente la caduta della marca preposizionale, bilanciata dall'introduzione dei trattini, con effetto di concisione e stilizzazione sintattica:

Mi stupì di sentire una magra ragazza - capelli a frangia, grosse labbra e sigaretta - gridare il nome della bruna del veglione (TDS 696).

Una ragazza - viso gonfio e capelli in disordine - vestito da sera di tulle celeste, senza scarpe (TDS 678).

Il fotografo - cravatta nera come Loris - si fregava le mani (TDS 743).

Padrino aveva allora il casotto di Gaminella - due stanze e una stalla - la capra e quella riva dei noccioli. (LF 782).

A vederla, era sempre la stessa - quegli occhi scuri scottanti (LF 866).

Ancora, in posizione incidentale si possono trovare sintagmi appositivi:

Quello della mattina, Nando, le manda gli altri (PT 32).

Ma uno, un giovanotto con la giacca sulle spalle, si stacca e mi dice: - Voi siete il macchinista? (PT 65).

Un biondo, Milo, prese lui la chitarra (CO 193).

C'era uno giovane, Giuseppe, che a suo padre gli avevano rotta la testa (CO 246).

Sono cose che un uomo, un cameriere, non farebbe (TDS 676).

Sembrava una città nuova, Torino, una città finita allora (TDS 680).

21 Affine a questa la costruzione nominale dove l'ellissi del verbo tematizza la ripresa pronominale: «Eravamo fermi sotto il sole che picchiava, lui col suo fazzoletto rosso e la barba di sei giorni» (PT 6); «Andavamo come i buoi senza sapere dove, lui col suo fazzoletto rosso al collo, il suo fagotto, le sue brache di fustagno» (PT 3). 
- Chissà l'ebanista di Palazzo Reale. Lo metterei a far palchetti, quel monarchico $(\operatorname{TDS} 757)^{22}$.

Avevo un musicante, Arboreto, che suonava il bombardino (LF 788).

Dopo Paesi tuoi la funzione apposizionale è demandata a incisi sintagmatici anche più lunghi:

L'oste, un omaccio coi peli nelle orecchie e il grembialone, ci si mise d'attorno (TDS 719).

Il sito era impossibile - un quartiere come a Roma non ce ne sono più, forse soltanto fuori porta (TDS 712) 23 .

talvolta sotto forma di enumerazioni bimembri o trimembri precedute o seguite da una clausola riassuntiva di registro colloquiale:

Se ne vedevano i frutti - quelle donne inferocite, quel ragazzo storpio (LF 799).

Un bel momento restiamo soli, io, Nando, Ernesto, sotto il cinghione teso, e gli altri erano tutti nella cucina e sulla porta (PT 84).

Ma adesso, polenta, coniglio e peperoni, tutto bruciava (PT 67).

E tutto, il sole la collina e la stalla, era pieno di mosche che mettevano sonno (PT 68). Nessuna di quelle vetrine e quelle insegne era dimessa e familiare come la ricordavo, non i caffè non le cassiere non le facce (TDS 680).

Capirei se dicessero i pennelli, i colori, l'aquaragia - le cose che adoperano - ma no, questa gente parla difficile solo per gusto (TDS 699).

Mi faceva un certo effetto rivedere le vecchie botteghe, i grandi portoni nelle viuzze, e leggere i nomi - delle Orfane, di Corte d'Appello, Tre Galline - riconoscendo le insegne (TDS 709) ${ }^{24}$.

In Paesi tuoi l'inciso elencativo può inserirsi in schemi frasali segmentati con dislocazione a destra, accrescendo la segmentazione della prosa:

Ci portò, me e Talino, all'osteria (PT 13).

Li lascio gridare, loro e le donne (PT 31).

Più raramente nei romanzi successivi, ma con un grado di elaborazione maggiore e l'uso di indicatori paragrafematici marcati:

Dopo un po' non c'era un pezzo - non una miniatura, non un manico d'ombrello - che non facesse gola di metterselo attorno o averci una casa e dargli un posto (TDS 728).

\footnotetext{
22 In apposizione è posto l'elemento tematizzato, dislocato a destra nella frase segmentata, dove il pronome ridondante riprende in anafora testuale il soggetto della frase precedente.

23 Il trattino all'inglese segnala qui l'intervento della riflessione dell'io narrante che comprende la situazione nuova confrontandola con esperienze precedenti.

24 In questo esempio, l'inciso evidenzia la riscoperta del nome come della concreta esistenza di cose per tanto tempo solo ricordate.
} 
Per un anno in più nessuno aveva fatto niente in campagna, e se tutti quegli uomini se ne fossero invece tornati a casa - i tedeschi a casa loro, i ragazzi sui beni sarebbe stato un guadagno (LF 803).

o con dilatazione del valore appositivo in enumerazione:

Ci si muoveva appena in mezzo alle montagne di roba - pezzi d'avorio, quadri scrostati, pendoli, statuine, fiori finti, collane, ventagli (TDS 728).

o in sequenze esplicative perfettamente bilanciate, che valgono a riequilibrare la forza frammentante dell'inciso:

Allora capii che la vera mamma, la sorella maggiore, la sorella esigente e cattiva, di Rosetta era costei, questa Momina che tirava le pietre e nemmeno nascondeva la mano - che, come Becuccio, non aveva più niente da perdere (TDS 766) ${ }^{25}$.

o a diluirla:

Era diviso in due parti da un'arcata, di qua le poltrone e i tavolinetti leggeri, di là un lungo tavolo lucido sotto un lampadario e una larga finestra a tre luci (TDS 764).

talvolta tramite il richiamo anaforico al segmento frasale precedente l'inciso:

Momina che mi stava raccontando quanto forte la prendesse a volte il disgusto non la nausea di questo o di quello, di una serata o di una stagione, ma lo schifo di vivere, di tutto e di tutti, del tempo che va così presto eppure non passa mai Momina accese una sigaretta e suonò il clacson (TDS 723).

Il ragazzo ci ascoltava appoggiato al muro, e mi accorsi che non era che ridesse aveva le mascelle sporgenti e i denti radi e quella crosta sotto l'occhio - sembrava che ridesse, e invece stava attento (LF 799).

Affine alla funzione apposizionale è quella di correctio:

Veniva notte - notte calda - e le piante fiorite facevano estate (CO 226).

Sapevo quel che un uomo - anche il migliore - può valere (TDS 691).

Venne del vino - vino nero, del fusto - qualcuna di noi prese la grappa (TDS 774).

Poi i pomeriggi d'inverno lo fecero entrare e lui, che girava con degli stivali da cacciatore, si batteva il bastoncino sullo stivale, si guardava intorno, strappava un fiore o un rametto nel giardino - meglio una foglia rossa di vite vergine - e saliva svelto la scala dietro i vetri (LF 860).

25 Si tratta di un inciso forte che spacca e moltiplica il sintagma genitivale: presa di coscienza da parte della protagonista della complessità della situazione. Dal secondo sintagma appositivo 'questa Momina' dipendono due relative restrittive, la seconda delle quali però dentro inciso - stacco logico forte nello sviluppo del ragionamento - e contenenente un secondo inciso comparativo. 
All'interno dei moduli propri dell'oralità assumono posizione parentetica la ripresa di una parola in epanadiplosi:

Ero stufo di vedere binari, stufo (PT 13).

Valla a prendere, va' (PT 43).

e in generale quegli «elementi di articolazione del discorso, che segnalano la frammentazione e il coinvolgimento caratterisitici della sintassi parlata» (Testa 1997: 172), tanto nelle parti diegetiche quanto in quelle mimetiche e più frequenti in Paesi tuoi dove Pavese si concentra sulla ricreazione del parlato popolare. Appellativi e vocativi:

In gamba, $\mathrm{Pa}$ ! (PT 18).

Donne, è arrivato! (PT 18).

Paura di cosa, zucca? (PT 18).

E' che non ci sono prove, caro voi (PT 64).

E per te, macchinista, cos'hanno piantato? (PT 31).

Non avevano trovato refurtiva neanche in fondo ai letti, villanzoni (PT 8).

Vigliacco e bastardo, sei soltanto capace a scappare di notte (PT 23) ${ }^{26}$.

Vecchio Vinverra, non te la cavi col vino (PT 29).

- Bisogna evadere, Rosetta. D'accordo? (TDS 716).

Chi aveva gli occhi spaventati e acquosi, poveretta, era la madre (TDS 715).

- È un bel pensiero per il suo risveglio. Ragazzaccio (TDS 722).

esclamazioni e imprecazioni:

- Diavolo, - fa il vecchio, saltando su, - l'avete legato il cane? (PT 25).

Quante botte, madonna, se fossi stato io Rico, quante botte (PT 65).

- Per carità. Purché niente succeda... (TDS 716).

interiezioni:

Ce n'è del lavoro in campagna, eh? (PT 19).

- Ah no, non ci truffi. Rispondi (CO 178).

Eh, Lubrani, l'hai trovata una con la testa sul collo (CO 183).

- Uh, - disse Rosetta, - svegliarsi è orribile... (TDS 733).

- Ah sì, - disse l'altra, indignata, - la colpa è delle madri (TDS 680).

- Ma sì, lo conosci... (TDS 720).

segnali discorsivi:

- Piuttosto, guarda, partiamo subito (PT 6).

- Senti, - gli dico, - se sono le ragazze che ti fanno gola, ti porto al buon indirizzo $(\text { PT } 7)^{27}$.

${ }^{26}$ In questo esempio e nel successivo ci troviamo di fronte a casi di monologo dell'io narrante.

${ }^{27}$ Per l'ammissibilità in posizione parentetica dei vocativi si vedano Serianni (2006: 73-74) e Cignetti (2001: 93-94) in particolare per la funzione di apostrofe; per la posizione parentetica dei segnali discorsivi, Bazzanella (1995). 
«Guarda, - pensai, - non si è fatto la barba nemmeno per lei» (CO 154).

Senti, - gli dissi una mattina, - hai avuto fortuna che ti sei fatto male tu (CO 157).

Senti, - mi disse, pigliandomi il braccio, - non far mai niente contro il Fascio, prometti (CO 185).

- E senta, l'ha vista, dopo? (TDS 686).

- Ecco, - disse Morelli, - arrivare (TDS 688).

- È un altro modo di guardar la cosa, se volete, non è un'altra cosa (TDS 700).

- Sentite. Non è meglio che ci diamo del tu? (TDS 707).

- Sai, il dramma finisce male... (TDS 717).

- Simpatica, vero, Rosetta (TDS 722).

In Tra donne sole poi, affinandosi e estendendosi le inserzioni di discorso indiretto, i segnali discorsivi in inciso vengono riportati anche tali quali nel discorso indiretto:

Non c'era niente di sporco, soltanto un'incoscienza - da bestie, se voleva, ma anche da gente inesperta che soltanto così possono capire chi sono (TDS).

In posizione incidentale sono poi le formule che introducono o concludono colloquialmente un argomento:

Basta, non tocca più a te (PT 86).

- So che l'hanno sgessato, - disse lei. - Che roba (CO 154).

- Stava già bene, nossignore, se la piglia col fascio (CO 184).

Io non so, meno mangia e più ingrassa (CO 186).

- Niente, - gli dissi. - Finiamo le casse (TDS 725).

- Sono stata ragazza, ecco tutto (TDS 754).

- Prendere il veleno a carnevale, che peccato (TDS 679).

In Tra donne sole anche nelle parti diegetiche:

Tanto, a Torino ci dovevo stare un pezzo (TDS 676).

Andammo a ballare al Nirvana. Nientemeno (TDS 757).

Becuccio non era militare, ecco tutto (TDS 759).

Tutto sommato, mi annoiai (TDS 729).

Tanto meglio, il va e il vieni della gente forestiera, la confusione e il baccano della piazza, avrebbero mimetizzato anche un negro (LF 785).

Annota Enrico Testa che «il tipo di narrazione autodiegetica, fondato sulla coincidenza tra io narrante e protagonista (ricorrente in quasi tutti i romanzi di Pavese, a esclusione del Carcere e della Bella estate), fa sì che le forme vernacolari e gergali», come quelle colloquiali e proprie dell'oralità, «si diffondano su tutti i piani del testo», diminuendo lo scarto tra la parte diegetica - che si configura come un racconto-monologo - e quella mimetica del dialogo tra i personaggi (Testa 1997: 233): l'occorrenza uniforme degli incisi di parola e sintagma si inserisce all'interno di questa direttrice stilistica. 
Solo nella Luna si assiste a una rarefazione di segnali discorsivi, vocativi e appellativi, sia perché si riduce lo spazio dedicato ai dialoghi, sia per l'approdo a un'estrema stilizzazione sintattica.

\section{Incisi didascalia}

La tipologia degli incisi inseriti come didascalie dei discorsi diretto e indiretto e dei monologhi del protagonista con se stesso è varia. Si va da un grado zero di assenza totale di introduttori e commenti:

- Si parla sempre della gente.

- Chi ne parla?

- Ma ieri Carletto. E quell'altro. Sempre.

- Non siamo mai noi due soli.

- Se sei qui stamattina. Ti dispiace? Vattene (CO 189).

- Non è nulla. Ho aspettato.

- Tutta la notte.

- Tutto l'inverno.

- Vuol dire che ha tempo (TDS 681).

al semplice inserimento di verba dicendi, a didascalie di respiro più ampio. Riguardo ai verba dicendi, sovente sono inseriti dopo la prima parola o il primo sintagma del turno dialogico, con effetto di forte segmentazione:

- Macché! - dice Talino - Sono bianche ch'è un piacere! (PT 11).

- Grane, - gli faccio, - grane e tabacco, questo sì (PT 31).

- Va bene, - le dico poi, mentre ci alziamo - allora vengo a prendere le camicie (PT 9).

- Sta bravo, Berto, - mi dice Talino, come se fossi il suo socio, - non hai lavorato neanche a Torino (PT 50).

- Al suo posto, - le dissi, - farei di tutto per levar di mezzo un Pablo. - Sciocco, - mi disse, - è per questo che deve aiutarti. Non mi faresti tu un piacere se io volessi?

- Lui lo sa, - disse poi, - che se riesci ti gira la testa e non pensi più a me $(\mathrm{CO}$ 192).

- Quel tuo amico, - fa Scarpa, - non hai chiesto ai comagni di qui? (CO 258).

- Storie, - disse Loris, - volevamo colorire un ambiente... (TDS 701).

- Era qui, - disse il primo. - Sarà in piazza -. Tornò a guardare nel bidone. - Va' a chiamarle Becuccio (TDS 689).

- No no, - disse Nuto, - la vince il parroco (LF 793).

Sovente, però, la spezzatura introdotta dalla didascalia è riequilibrata dalla ripresa ad anello del sintagma precedente l'inciso:

- Bella roba, - m'interruppe sarcastico, - bella roba (TDS 713). 
- E lei, - disse Febo, sempre guardando impertinente in sala, - lei è uscita dal suo ambiente, si è messa le calze di seta e con noi gente per bene e istruita si diverte (TDS 738) ${ }^{28}$.

- Ma ce l'ho, - disse Momina gridando. - Ce l'ho, la pagnotta. Che cosa posso farci se ce l'ho? (TDS 767).

- Ce n'è, - disse, - ce n'è. (LF 803 battuta pronunciata da Valino) ${ }^{29}$.

mentre in Paesi tuoi e nel Compagno introduttori e commenti ipertrofici incorniciano battute di dialogo che appaiono quasi per inciso:

Quando ci siamo tutti e tre, il vecchio mi dice: - Al piacere -, e tracanna e poi butta la goccia per terra (PT 21).

Le dicevano: - Sedetevi, Ma'-, perché chinandosi gemeva e aveva sempre qualcuno nelle gambe (PT 22).

Linda gli disse: - Vieni presto -, con l'allegria che hanno le donne, poi si cacciò tra le coperte (CO 187).

Ogni pezzo finito, dicevano «Bravo» e Lubrani allungava il bicchiere (CO 180).

Vi sono poi i casi di didascalie a battute di dialogo che il protagonista immagina di pronunciare:

- Senti l'odore della stalla? - volevo chiedergli, ma lui si godeva fin il fresco della corsa tant'era contento (PT 17).

In quei momenti avrei potuto dirle: «Andiamo a far l'amore» che sarebbe venuta (CO 191).

Com'è il mondo, pensavo, una volta avrei detto «Salute» (CO 205).

- Tu fai la fame, cara te, - volevo dirle, - e hai tante storie (TDS 701).

Talvolta è la risposta immaginata e non pronunciata che funge da didascalia alla battuta di un personaggio:

E mi fa: - Cosa direbbe Pieretto se sapesse! - Direbbe che sei sempre la stessa, penso io [...] Ebbe ancora il coraggio di dirmi: - Se Pieretto sapesse!... Se Pieretto sapesse gli rincrescerebbe più per me che per te (PT 10).

Talvolta la risposta a una battuta di dialogo è riportata in discorso indiretto, seguita da didascalia:

- Che amica?

Quelle due del veglione - l'avevo capita (TDS 692).

Sono poi frequenti le didascalie al discorso indiretto:

Già una volta, mi disse, s'era lasciata la mano libera alle masse (CO 270).

Miserie, mi disse, più nessuno pagava l'affitto (TDS 710).

E una volta, dicevano i vecchi, era stato ancora peggio (LF 844).

28 Qui la ripresa pronominale è inserita in parallelismo trimembre.

29 Strutture a anello incorniciano anche altri tipi di incisi: «Accamparsi e dormire in quel deserto donne e bambini - in quel deserto ch'era casa loro, dove magari coi serpenti s'intendevano» (LF 820). 
e al monologo che il protagonista rivolge a se stesso:

Guardali bene, Berto, dico senza fermarmi, è in mano a questa gente che ti sei messo (PT 13).

Stiamo attenti, non siamo più a Torino, dico tra me in quel caldo (PT 22).

Siamo in mezzo a due mammelle, dico: qui nessuno ci pensa, ma siamo in mezzo a due mammelle (PT 24, con foderamento).

«Stai zitto, - pensavo, - stai zitto» (CO 188).

«Sono a Roma» dicevo tra me, «Sono a Roma» (CO 224).

- Il mondo è grande, - dissi forte, senza saper bene perché (TDS 690).

$\mathrm{Ma}$ - qui Morelli non aveva torto - nonostante tutto ero tenuta a ringraziare quei giorni (TDS 691).

Vi sono infine incisi che svolgono una funzione simile a didascalie: sono i commenti del protagonista alla narrazione che va svolgendo:

Non aveva mai attraversato un corso, si vede, o mi stava già lavorando (PT 3).

Eravamo saliti bene, perché di là si vedeva, scommetto, fino a Torino (PT 34).

Per lavorare, lo sapevo, bisognava darsi attorno (CO 193).

La bionda stessa ci guardava imbambolata e, mi parve, delusa (TDS 692).

E quello, si capisce, voleva convincerlo (TDS 696).

I nostri amici, per fortuna, s'erano da un pezzo dispersi nelle sale da gioco (TDS 716).

Rosetta mi guardava intimidita, dal fondo degli occhi - mi parve un'altra in quel momento - e bisbigliò: - Dopo si sta peggio che prima (TDS 718).

Le dissi che ai tempi ch'ero stata innamorata, per quanto capissi benissimo - queste cose si sanno - ch'eravamo due matti, che il mio uomo era un incapace, che se ne stava in casa a dormire mentr'io correvo per Roma, malgrado tutto questo, non s'impara a bastar da soli se non s'è fatta l'esperienza in due (TDS 754: inciso primario dentro inciso secondario che si dispone su tre membri equivalenti risolti in una formula riassuntiva).

Queste cose piacevano - salvo a Nuto, si capisce, che cercava lui di capir me (LF 814).

Le didascalie in sostanza danno spazio agli 'a parte' dell'io narrante a ogni livello del racconto: «la tendenza monologante finisce per intaccare [...] tutto il tessuto della scrittura»: da qui la «ricreazione fantastica del dialetto e della lingua parlata» (Mutterle 1970: 581-583) nella quale tanta parte hanno gli incisi.

\section{Incidentali di secondo grado}

Le incidentali di secondo grado giocano un ruolo fondamentale soltanto in Paesi tuoi, innervando appunto la prosa di questo romanzo del capillare radicamento dell'io dentro i fatti che sta raccontando, delle sue percezioni e dei suoi giudizi, intera- 
gendo di frequente con i modi scorciati e ellittici dell'oralità, come nel caso di costruzioni causali quali (Renzi 2001: 789):

Faceva ridere la vecchia che, sdentata com'era, si succhiava i pomodori (PT 23). Penso che, goffo com'era, era riuscito proprio a farmela (PT 25).

Tracagnotta com'era, faceva rabbia (PT 47).

L'aria cruda mi mordeva le gambe e, stanca com'ero, indugiavo davanti alle vetrine (TDS 676).

Mi faceva rabbia che sapesse tante cose, giovane com'era, che ci scherzasse così, che per lui fossero come dei denari che si era trovato in tasca (TDS 727) ${ }^{30}$.

o nel caso di subordinate implicite frapposte tra soggetto e verbo della principale, di registro colloquiale:

Poi l'Adele, mangiato e bevuto, va per la strada di Gisella (PT23).

Gisella, lavata e vestita, poteva fare la sua figura anche a Torino (PT 53).

o con soggetto diverso da quello della principale, di registro letterario, sul tipo dell'ablativo assoluto latino:

Partiti tutti dal cortile, non mi ero mosso e avevo visto le donne salire e scendere la scala, e la vecchia tagliare col filo la polenta e darne ai bambini, e poco alla volta s'era fatto silenzio (PT 80).

Venne il momento che, tornando il cameriere con un nuovo vassoio, riaccendemmo la luce (TDS 733).

Andò che, telefonandomi Momina, le dissi che Mariella aveva ragione (TDS 755).

L'insistenza un po' ingenua sul punto di vista dell'io narrante sviluppata da incidentali primarie e secondarie accentua in Paesi tuoi l'aporia tragica che è il vero centro del romanzo: un episodio della vita di un uomo che è manovrato e mosso da un destino che non comprende finché non si compie. Lo spessore dei personaggi e lo scavo continuo nelle "ragioni e irragioni del fatto" 31 marcano la distanza tra Paesi tuoi e la ventura poetica neorealista.

Ma dopo Paesi tuoi Pavese affina la tecnica della narrazione autodiegetica riducendo la contaminazione del registro popolare e colloquiale sul lessico e sulla sintassi (diversa del resto è anche l'ambientazione degli altri romanzi), come si è accennato più sopra: in particolare per le subordinate implicite in inciso Pavese preferisce l'uso di grado zero con soggetto uguale alla principale:

\footnotetext{
${ }^{30}$ In questo esempio, l'inciso è parzialmente riassorbito nel parallelismo.

${ }^{31}$ La carenza di questo 'ingrediente’ è rimproverata da Gadda alla narrativa neorealista: «Il dirmi che una scarica di mitra è realtà mi va bene, certo; ma io chiedo al romanzo che dietro questi due ettogrammi di piombo ci sia una tensione tragica, una consecuzione operante, un mistero, forse le ragioni o le irragioni del fatto [...]. Vorrei, dunque, che la poetica dei neorealisti si integrasse di una dimensione noumenica, che in alcuni casi da me considerati sembra alquanto difettarle», cit. in Corti (1978: 36).
} 
Così scherzando, mi schizzò il progetto di una casetta di vetro (TDS 712).

Bevendo grappa e mangiando salsicce, si riparlò della montagna (TDS 715).

M'accorsi che ero molto più sola a Roma, arrampicandomi per quelle strade o entrando a prendere il caffè da Gigi, che non a Torino nel mio letto d'albergo o in via Po (TDS 726).

Mi guardò con un occhio solo, ridendo (TDS 713).

Altre volte relative descrittive o subordinate in funzione circostanziale aggiungono alla scena o ai personaggi i particolari colti dall'occhio del protagonista:

Solo il fratello di Damiano, ch'è il più giovane, mi domandò se aveva insieme una bionda (PT 7).

Momina, che aveva acceso una sigaretta, mi squadrò con gli occhi socchiusi nel fumo (TDS 708).

E io, che avevo visto Talino là dentro fare il bagno, mi figuravo che pelle e che sudore dovevano averci tutte sotto la camicia (PT 23) 32 .

Sembrava Torino quando si esce prima di giorno, che non ci sono neanche i tranvai (PT 28) ${ }^{33}$.

Faceva un gran caldo, tanto che sudavo tutto, per via della cravatta stretta (PT 3). Finché la vita aveva un bagno, valeva la pena di vivere (TDS 677).

Io, se non dovessi lavorare, avrei dei vizi terribili (TDS 740).

Allora mi guardò con un occhio solo, come aveva fatto uscendo di là dentro, e a me veniva la rabbia (PT 5).

Sono frequenti però dopo Paesi tuoi i casi in cui le incidentali secondarie o vengono evidenziate da indicatori marcati:

Io guardavo e cercavo di capire la materia e il segreto - come si fa per un modello - ma era più che difficile inutile (TDS 729).

o vengono indebolite e allineate in sequenze bimembri:

Mi colpì che rideva in dialetto, come ridono le commesse, come rido qualche volta anch'io (TDS 699).

o, anche nel discorso indiretto, riassorbite da sequenze anaforiche:

Le dissi che la colpa era sua; che, se anche Rosetta non si ammazzava, la colpa era sua. Le dissi non so che cosa (TDS 777).

${ }^{32}$ L'inciso qui convive con elementi del registro non letterario: l'eufemismo gergale, il piemontesismo figurarsi, l'interrogativa indiretta con indicativo imperfetto.

${ }^{33}$ La proposizione parentetica, nell'esempio, è introdotta da un che polivalente. 


\section{Conclusioni}

In conclusione, l'analisi evidenzia una distanza piuttosto marcata tra Paesi tuoi e i romanzi successivi (già notata anche a livello lessicale, non solo sintattico (Beccaria 1989: 85), dove Pavese abbandona quell'elementarità un po' ingenua che adeguava la scrittura «alla quotidianità dei contenuti e alla semplicità dei rapporti»e al tempo stesso filtrava il contenuto, «allontanandolo da una dimensione realisticoanalitica» (Matarrese 1973: 290). Alla preoccupazione di una resa «naturalistica» della realtà subentrano in Pavese, soprattutto dopo il Compagno, istanze diverse quali l'approfondimento delle potenzialità della narrazione autodiegetica e la necessità di un approdo a un «grande stile» che viene «da molto lontano» e rimane equidistante «da ogni tipo di prosa che abbia il grado zero della naturalezza discorsiva, della freschezza convenzionale, o il grado plurimo del libresco, del prezioso, dell'allusivo» (Beccaria 2000: XXVI): equidistante cioè tanto da un dettato che tenda univocamente alla «frammentazione» e alla «stilizzazione» quanto da un fraseggio votato unicamente alla «complessità ed espansione periodale» (Beccaria 2000: ivi).

Negli ultimi romanzi le incidentali primarie con introduttori grafici marcati sono così pervasive del tessuto sintattico da indurre a riflettere sulla connessione tra queste e il carattere autodiegetico della narrazione. Se senz'altro alle incidentali è demandato il compito di moltiplicare i piani discorsivi e dunque corrugare tanto il livello semantico quanto quello linguistico del racconto, proprio la diffusione capillare nel testo le sgancia dal loro caratteristico incarico di 'a parte' del narratore facendone uno strumento sintattico maneggevole e polifunzionale. Per Pavese, funzionale soprattutto a una resa sintattica veloce, segmentata, ellittica ma mai caotica in quanto organizzata sulla trama di una oralità stilizzata e sull'ordito di simmetrie e parallelismi di stampo classico.

\section{Bibliografia}

ANTONELli, Giuseppe (2008): «Dall'Ottocento a oggi», Bice Mortara Garavelli (a cura di), Storia della punteggiatura in Europa. Roma-Bari, Laterza.

Asor RosA, Alberto (2003): «La storia del «romanzo italiano»? Naturalmente, una storia anomala», in F. Moretti, P. V. Mengaldo, E. Franco (a cura di): Il romanzo. Storia e geografia, vol. III. Torino. Einaudi, pp. 255-306.

Bazzanella, Carla (a cura di) (1995): «I segnali discorsivi», in L. Renzi, G. Salvi, A. Cardinaletti, Grande Grammatica Italiana di consultazione. Bologna, Il Mulino, vol. III. pp. 223-257.

BECCARIA, Gian Luigi (1989): Le forme della lontananza. Milano, Garzanti.

BECCARIA, Gian Luigi (2000): «Introduzione» in C. Pavese, La luna e i falo. Torino, Einaudi, pp. V-XXXIII.

Borgato, Gianluigi, SALVI, Giampaolo (1995): «Le frasi parentetiche», in L. Renzi, G. Salvi, A.

Cardinaletti, Anna (a cura di) (2001): Grande grammatica di consultazione. Bologna, Il Mulino, vol III, pp. 165-174.

CANE, Eleonora (1969): Il discorso indiretto libero nella narrativa italiana del Novecento. Roma, Silva. 
CignetTI, Luca (2001): «La [pro]posizione parentetica: criteri di riconoscimento e proprietà retorico-testuali», Studi di grammatica italiana, XX, 2001, pp. 69-125.

ColetTI, Vittorio (1989): "Con voce più alta e stizzosa. Osservazioni su discorso diretto e dialogo nel romanzo», in Italiano d'autore. Saggi di lingua e letteratura del Novecento. Genova, Marietti, pp. 43-55.

Coletti, Vittorio (2003): «La standardizzazione del linguaggio: il caso italiano», in F. Moretti, P. V. Mengaldo, E. Franco (a cura di): La cultura del romanzo. Torino, Einaudi, vol. I, pp. 309-346.

CoRTI, Maria (1978): Il viaggio testuale. Le ideologie e le strutture semiotiche. Torino, Einaudi.

DARDANO, Maurizio (1992): Studi sulla prosa antica. Napoli, Morano.

Devoto, Giacomo (1950): Studi di stilistica. Firenze, Le Monnier.

FERRARI, Angela, ZAMPESE, Luciano (2000): Dalla frase al testo. Una grammatica per l'italiano. Bologna, Zanichelli.

Grassi, Corrado (1964): «Osservazioni su lingua e dialetto nell'opera di Pavese», Sigma, I, 3-4, pp. 49-71.

LONZI, Lidia (1981): «Avverbi frasali e strutture parentetiche», Lingua e stile, XVI, pp. 393431.

MATARRESE, Sabatina (a cura di) (1973): «Il primo Pratolini fra tradizione toscana, prosa solariana e ermetismo», in P. V. Mengaldo, Profili linguistici di prosatori contemporanei. Padova, Liviana, pp. 243-305.

Mengaldo, Pier Vincenzo (1992): Il Novecento. Bologna, Il Mulino.

Mengaldo, Pier Vincenzo (1996): Alcuni problemi della prosa contemporanea, in Id., La tradizione del Novecento. Prima serie. Torino, Bollati Boringhieri, pp. 163-173 (il saggio era apparso in introduzione al volume Profili linguistici di prosatori contemporanei. Padova, Liviana, 1973, riedito nella prima edizione della Tradizione del Novecento.Milano, Feltrinelli, 1975).

Mortara GARAVELli, Bice (1956): Studi sintattico-stilistici sulle proposizioni incidentali. Torino, Giappichelli.

Mortara Garavelli, Bice (2003): Manuale di retorica. Milano, Bompiani.

Mutterle, Anco M. (1969): «Pavese 1936-1938», Comunità, XXIII, pp.122-143.

Mutterle, Anco M. (1970): «Ciau Masino: dal plurilinguismo al monologo interiore», Belfagor, XXV, 5, pp. 559-591.

Mutterle, Anco M. (1998): «Rileggendo Pavese», Studi Novecenteschi, XXV, 56, pp. 179204.

PAVESE, Cesare (2000): Tutti i romanzi, a cura di Marziano Guglielminetti. Torino, Einaudi.

PAVESE, Cesare (2000b): Il mestiere di vivere, a cura di Marziano Guglielminetti e Laura Nay, nuova introduzione di C. Segre. Torino, Einaudi.

PÉTILlON-Boucheron, Sabine (2002): Les détours de la langue. Étude sur la parenthèse et le tiret double. Louvain-Paris-Dudley (MA), Éditions Peters.

SERIANNI, Luca (2006): Grammatica italiana. Italiano comune e lingua letteraria [1989], con la collaborazione di A. Castelvecchi. Torino, UTET.

Tellini, Gino (1998): Il romanzo italiano dell'Ottocento e Novecento. Milano, Bruno Mondadori.

Telve, Stefano (2008): L'italiano: frasi e testo. Roma, Carocci.

TeStA, Enrico (1997): Lo stile semplice. Discorso e romanzo. Torino, Einaudi. 